\title{
Eyes Don't Lie: Understanding Users' First Impressions on Websites Using Eye Tracking
}

\author{
Hong Sheng, Nicholas S. Lockwood, and Sirjana Dahal \\ Missouri University of Science and Technology, MO, USA \\ \{Hsheng, lockwoodn\} @mst.edu, dahalsirjana@gmail.com
}

\begin{abstract}
Websites are prevalent these days. Web users make instantaneous judgments regarding a website based on their first impressions and usually decide either to stay on the website or bypass it during their initial interaction with the website. Hence, understanding users' first impression is important for both practitioners and researchers. This research examines users' first impression of websites using an eye tracker. Eye tracking can provide fixation points where users focus their attention on a stimulus. The eye tracking results indicate that users take at least 2.66 seconds to scan the website before they fixate their eyes on an element of the website. Our analysis also shows that first fixation duration lasted for 180 milliseconds. This indicates that after allocating attention to a specific area on a website, the eyes stopped to focus for 180 milliseconds during which the brain processed the visual information received from the eyes to organize the information and form an impression of the website.
\end{abstract}

Keywords: First impression, Websites, Eye Tracking, Attention.

\section{Introduction}

Websites are pervasive and commonplace these days, so they need to create a favorable first impression on users during initial exposure (Thomson, 2006). Web users make instantaneous judgments regarding a website based on their first impressions and usually decide either to stay on the website or bypass it within the first couple of minutes (Thilmany, 2003).

First impressions are powerful and often have a long-term effect on users' perceptions and attitudes towards a website. Therefore, it is critical for the designers, web developers, and organizations to understand how users form their first impressions of websites. In this study, we use an eye tracker to track and analyze users' interactions with various websites during their initial exposures, which shed light on the time needed to form first impressions as well as the processes involved in selecting and organizing relevant information to form first impressions.

\section{Literature Review}

First impressions have been studied in various contexts such as psychology, medicine, usability, marketing, etc. (Lingaard et al., 2006). In marketing, first impression is 
defined as a quick evaluation made by the customer during the first few minutes of an encounter with a product/object (De Groot, 2006). Consumers' impressions usually remain stable (Mitchell and Corr, 1998) and have long-term effects, sometimes referred to as the 'halo effect'.

In examining a person's perception of another, Hamilton et al. (1980) define impression as a perceiver's cognitive representation of another person. They focused their inquiry on the cognitive processes involved in the development of that cognitive representation from the stimulus information available to the perceiver (Hamilton et al., 1980). Willis and Todorov (2006) further concluded that a minimal exposure time of 100 milliseconds is sufficient for a person to make a specific trait inference of others, such as attractiveness, trustworthiness, likeability, competence, and aggressiveness, based on facial appearance.

In the decision-making literature, first impression is referred as a cognitive confirmation bias (Lingaard et al., 2006). Confirmation bias occurs when participants who have formed positive first impressions may ignore negative issues and errors that they encounter later. Likewise, participants find it hard to accept the positive information when negative first impressions have been formed. Lindgaard et al. (2006) conducted a study to determine the exposure time required for the participants to form first impressions and concluded that users take 50 milliseconds to make a decision on whether they like or dislike what they see.

According to Hamilton et al. (1980), forming an impression is an active process in which the perceiver organizes the information available about a target to develop a coherent representation of that target. Fiske and Neuberg (1990) proposed a continuum model of impression formation in which attention to and interpretation of information underlies the impression formation process. Attention is a selective process that allows us to prioritize and focus on certain areas or aspects of a visual stimulus while ignoring others (Canasco, 2011). Perceivers allocate attention to the target if the target is of at least minimal interest (Fiske and Neuberg, 1990). Once this information is processed, it is organized into a cognitive representation which leads to impression.

\section{$3 \quad$ Research Methodology}

This research is an exploratory examination of users' first impressions of websites. An eye tracker was used in collecting users' eye movements during the exposure of the websites, which provided insights into users' impression formation process.

Twenty students from a mid-sized Midwestern university participated in this study. The participants all had normal vision with no color blindness. Among the 20 participants, 15 were male and 5 were female, with ages ranging from 19-36. They all had substantial Internet experience.

In this study, twenty-five websites from the top five tiers of university ranking of the Unites States that offer Law as a graduate or undergraduate degree were selected. These websites vary in levels of visual appeal and design features. Compared to other public websites, these websites receive less public exposure and traffic, which is appropriate for understanding users' first impressions. 
The study was conducted in the laboratory setting with each session lasting for approximately 60 minutes. Upon arrival at the lab, the participants were briefed about the experiment and eye tracking equipment. They were then asked to sign a consent form. Before the start of the experiment, each individual participant's eyes were calibrated using the eye tracker. A short questionnaire was also provided to collect the participant's demographic information. The stimulus was then presented on the eye tracking monitor to collect data on the participant's eye movements and fixation points. The twenty-five websites were displayed in random order. The participants were asked to view each website until they have formed their first impressions on the website and then move on to the next website. No time constraint was imposed. After viewing each website, the participants were then asked to rate each website based on their first impressions. An qualitative interview was conducted at the end of each session.

A Tobii 1750 eye tracker system was used to capture participants' eye movements and fixation points. The Tobii 1750 eye tracker-which functions without the need for glasses, lenses, or headgear — can gather eye tracking data without intruding and interfering with participants' performance on assigned tasks.

\section{$4 \quad$ Results and Discussion}

\subsection{Time to First Fixation}

Human eyes are constantly moving until they stop and focus on a point. When the eyes stop to focus, it is called a fixation (Technology, 2011). The length of the stops, when the eyes fixate, varies from about 100 to 600 milliseconds depending on what the eyes are looking at. During this stop, the brain starts to process the visual information received from the eyes (Rayner, 1998). Fixations are important because they locate the foveal vision. Users cannot interpret what they have seen until they pay attention to it or fixate their eyes on it (Wedel and Pieters, 2007). Hence, the length of fixation is an indication of information processing and cognitive activities.

Time to first fixation is the time in seconds from when the stimulus was shown until the start of the first fixation within a page. In this study, time to first fixation was extracted from the eye tracker by considering each website as an area of interest (AOI). The descriptive statistics shows a mean of 2.66 seconds, which means that it took an average of 2.66 seconds for the participants to allocate their attention to the webpages.

\subsection{First Fixation Duration}

First fixation duration is the duration of the first fixation on an image regardless of whether it is the only fixation or the first of multiple fixations on an image (Technology 2011). First fixation duration was extracted from the eye tracker for all twentyfive websites. Average duration of the first fixation is 180 milliseconds.

Our analysis shows that first fixation duration lasted for 180 milliseconds. This indicates that after allocating attention to a specific area on a webpage, users' eyes stopped to focus for 180 milliseconds on the area. During this time, the brain 
processed the visual information received from the eyes to organize the information and form a cognitive representation of the website. This suggests that at least 180 milliseconds are needed in order for a user to form a first impression of a website, contrary to prior findings that first impression only takes 50 milliseconds to form (Lindgaard et al., 2006).

Table 1. Impression Rating Dimensions

\begin{tabular}{|l|l|l|}
\hline Item & \multicolumn{1}{|c|}{ Negative Anchor } & \multicolumn{1}{c|}{ Positive Anchor } \\
\hline 1 & Boring & Interesting \\
\hline 2 & Irritating & Charming \\
\hline 3 & Pale & Vibrant \\
\hline 4 & Complex & Simple \\
\hline 5 & Inconsistent & Consistent \\
\hline 6 & Awkward & Straightforward \\
\hline 7 & Cluttered & Clear \\
\hline 8 & Unreliable & Reliable \\
\hline 9 & Unattractive & Attractive \\
\hline 10 & Unappealing & Appealing \\
\hline 11 & Brief & Lasting \\
\hline 12 & Emotionless & Soulful \\
\hline 13 & Unpleasant & Pleasant \\
\hline 14 & Insignificant & Significant \\
\hline 15 & Pacifying & Exciting \\
\hline 16 & Abominable & Likable \\
\hline 17 & Poor & Rich \\
\hline 18 & Conservative & Progressive \\
\hline 19 & Dull & Witty \\
\hline & & \\
\hline
\end{tabular}

\subsection{Impression Perceptions}

Participants were asked to rate their impressions of each website along the nineteen semantic differential dimensions shown in Table 1 . The items were coded on a -3 to +3 scale and aggregated to obtain a single overall measure of first impression valence. Thus negative scores indicated unfavorable impressions and positive scores indicated favorable impressions. Sixteen of the stimulus websites were found to elicit favorable impressions on average and nine were found to elicit unfavorable impressions.

\subsection{Total Fixation Duration}

An independent samples t-test was conducted to compare the mean total fixation duration of participants between the favorable and unfavorable websites. Based on the results shown in Table 2, we see that there is a significant difference between the two groups $(\mathrm{p}=0.026)$. The mean values shown in Table 3 indicate that participants spend more time on favorable websites than unfavorable websites. 
Table 2. Comparison of Total Fixation Duration between Favorable and Unfavorable Websites

\begin{tabular}{|c|c|c|c|c|c|c|c|}
\hline & \multicolumn{7}{|c|}{ t-test for Equality of Means } \\
\hline & \multirow[b]{2}{*}{$\mathrm{t}$} & \multirow[b]{2}{*}{ df } & \multirow[b]{2}{*}{ Sig. } & \multirow{2}{*}{$\begin{array}{l}\text { Mean } \\
\text { Difference }\end{array}$} & \multirow{2}{*}{$\begin{array}{l}\text { Std. Error } \\
\text { Difference }\end{array}$} & \multicolumn{2}{|c|}{$\begin{array}{l}\text { 95\% Confidence Interval of } \\
\text { the Difference }\end{array}$} \\
\hline & & & & & & Lower & Upper \\
\hline $\begin{array}{l}\text { Total Fixation } \\
\text { Duration }\end{array}$ & 2.380 & 23 & .026 & 2.20681 & .92736 & .28841 & 4.12520 \\
\hline
\end{tabular}

Table 3. Mean Values of Total Fixation Duration

\begin{tabular}{|l|l|r|r|r|}
\hline & \multicolumn{1}{|c|}{ Websites } & \multicolumn{1}{c|}{ N } & Mean & Std. Dev. \\
\hline \multirow{2}{*}{$\begin{array}{l}\text { Total Fixation } \\
\text { Duration }\end{array}$} & Favorable & 16 & 19.991 & 1.924 \\
\cline { 2 - 5 } & Unfavorable & 9 & 17.784 & 2.701 \\
\hline
\end{tabular}

\subsection{Qualitative Data Analysis}

The qualitative analysis identified various issues with the website design and also revealed a number of ways in which the website design can be improved to affect first impressions. The responses from participants were analyzed, coded, and categorized with reference to design factors identified by various researchers (Fitneva et al., 2010; Cyr et al., 2006; Rayner, 1998; Zhang, 2000). The participants were shown the gaze plots and asked questions regarding the pattern of their fixation. They were also asked to provide recommendations on the design of the website. The responses were coded and categorized as below:

Colors. The first design factor identified by participants was use of color. Pacifying and relaxing use of colors such as light green, light blue, and yellow were preferred and the use of colors such as bright red, dark blue, and black were not preferred by the participants. Participants recommended the main color and background color to be pleasant and attractive and the contrast of the text color should be such that it is easier to read. One of the participants stated, "The use of three colors like blue, red and white reminds me of an airline website rather than website of a university." Another participant stated, "The use of background color as light blue and text color as white makes it harder to read the text."

Images. Another design factor identified by participants was the use of images. Participants suggested that use of meaningless and irrelevant pictures by some of the websites led to negative impression on those websites. Participants recommended that the main image has to be professional and related to the university. The website should not be cluttered with too many images either. One of the participants stated, "Too many images have made the website cluttered. They could have used a flash player for all the images and animated them." Another participant stated, "Big image on the home page gives me good impression." 
Navigation. One of the most common design factors identified by the participants was navigation. Navigation can further be classified into navigation on the main menu, left navigation, right navigation, top navigation, and bottom navigation. A majority of the participants suggested that the main menu be located on the top or left of the website drew more attention to the website since they know where to navigate the website. Too many navigation links on the bottom and right of the website led to confusion and negative impressions toward the website. One of the participants stated, "Menus on menus does not look attractive. The sub menus should be hidden within the main menus."

Text. Another design factor identified by the participants was text, which includes font size and style. Use of irregular text size and fonts were an issue to a few participants. Most of the participants suggested that the font type and size used on the websites be clear and readable. Some of the websites that used too much text on an image or unreadable text due to bad contrast of the color with the background led to negative impression of the users towards the website.

Position. Sequential position of images and text on the website were identified as other design factors that led to the formation of impressions towards that website. Participants suggested that use of a large and meaningful image on the area where eyes hit the website leads to the formation of a positive impression. Websites that are cluttered with too many images, links, and text do not capture attention toward the website. One of the participants stated, "This website is complete pain to navigate because of the weird position of images and texts. It looks like an advertisement of a product rather than a website of a university."

Space. Proper use of white space is also important. One problem identified on the unfavorable websites was the improper use of white space. Participants recommended that the websites need to make good use of white space by providing the right content. The websites with too much white space are repulsive since they provide very little content.

\section{Conclusion}

This study examined initial impression formation of twenty-five different university law school websites. Participants were asked to view each website with no exposure time limitation and their eye movements were tracked during the interaction.

The eye tracking results indicate that users take at least 2.66 seconds to scan the website before they fixate their eyes on an element of the website. Our analysis also shows that first fixation duration lasted for 180 milliseconds, which indicates the time needed for the brain to process the visual information received. In addition, the total fixation time was greater for websites that received generally favorable impressions than for websites that received generally unfavorable impressions. 
A follow-up qualitative analysis highlights key website features that participants noticed when forming their first impressions. Positive impressions were formed when websites made use of relaxing colors, relevant and professional images, simple navigation, readable text, well-positioned elements, and proper use of white space.

\section{References}

1. Hamilton, D., Katz, L., Leirer, V.: Cognitive representation of personality impressions: Organizational processes in first impression formation. Journal of Personality and Social Psychology 39(6), 1050-1063 (1980)

2. Lindgaard, G., Fernandes, G., Dudek, C., Brown, J.: Attention web designers: You have 50 milliseconds to make a good first impression! Behaviour \& Information Technology 25(2), $115-126(2006)$

3. Thomson, A.: Does your website attract or detract? Risk Management 53, 50 (2006)

4. Thilmany, J.: Web search measure website appeal in seconds. Mechanical Engineering $125,10-14$ (2003)

5. De Groot, N.: Importance of first impressions. American Book Publishing, Salt Lake City (2006)

6. Mitchell, M., Corr, J.: The first five minutes: How to make a great first impression in any business situation. Wiley (1998)

7. Willis, J., Todorov, A.: First impressions: Making up your mind after a 100-ms exposure to a face. Psychological Science 17(7), 592-598 (2006)

8. Fiske, S., Neuberg, S.: A continuum model of impression formation: From category-based to individuating processes as a function of information, motivation, and attention. Advances in Experimental Social Psychology 23, 1-74 (1990)

9. Technolog. Eye tracking as a tool in package and shelf testing, http: / / www . tobii.com/Global/Analysis/Training/WhitePapers / Tobii_EyeTracking_in_Package_and_Shelf_Testing_WhitePaper.pdf (accessed on: October 25, 2011)

10. Rayner, K.: Eye movements in reading and information processing: 20 years of research. Psychological Bulletin 124(3), 372 (1998)

11. Wedel, M., Pieters, R.: Informativeness of eye movements for visual marketing: Six cornerstones. In: Visual Marketing: From Attention to Action, pp. 43-71 (2007) 